\title{
Theory of Shadow
}

\author{
Prabin K.C. \\ Tribhuvan University, Prithwi Narayan Campus, Pokhara \\ Post Box No-316, Pardi-17, Birauta, Email: Pkmiracle36@gmail.com
}

\begin{abstract}
Generally, the opposite nature of brightness is supposed to be darkness i.e. shadow. For the formation of shadow, source of light; object and screen are required. We can see forming shadow, but we can not see the some rules of nature that governs the shadow. As for example- $F=M A$. This is the famous equation from the Newton's second law of motion. It shows the relationship among applied force $F$, mass of body $M$ and acceleration produced on body A. This even helps to formulate the much famous equation $E=M C^{2}$. In the same way, 'Theory of shadow' also shows the relationship among the different factors. From the close observation, when the symmetrical point source is taken, object distance; area of object and shadow distance are interrelated to the nature and size of shadow. 'Theory of Shadow' focuses on how these different factors are interconnected, how do they rule the formation of shadow and finally give the whole conclusion in the beauty of equation. For this work, experimental; mathematical and geometrical procedures are being applied while in each and every trial, The 'Theory of Shadow' is found to be exactly proved
\end{abstract}

Key words: Point source, area of object; object distance; area of shadow and shadow distance etc.

\section{Introduction:}

Point source is the source whose the size is equivalent to the wave length that the source radiates. Point source of light is the symmetrical source of light which emits light energy in all direction uniformly and equally. As like the point charge in Gaussian's theorem, the point source of light is used in developing the theory of shadow.In general, the point source of light is the spherical point having radius equals to zero, emitting rays of light uniformly in all direction from point.

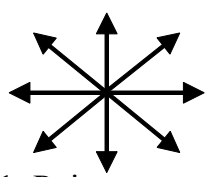

Fig 1: Point source of light

Electrical bulbs, candle, fluorescents lamps etc are not point sources of light. In these sources of light, rays of light do not radiate from a single point being concentrated on that point but from many points. Let us describe very simple method of designing a model of point source, any source of light like electrical bulb and Card board are taken. Small hole is created in the card board through which rays of light can pass. When the light is illuminated on the card board by electrical bulb, hole made in card board acts as a point source of light.
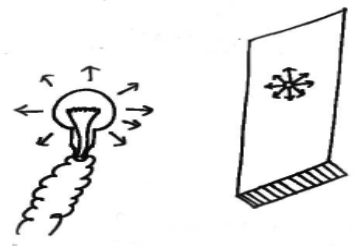

Fig 2: small hole made in card board acts as point source of light.
In Gaussian's theorem, when point source is enlarged, charged sphere is used for various purposes. Similarly, in course of shadow theory, the bigger size of source of light is also the spherical source of light where radius (r) equals not to zero. Such spherical source of light with non zero radius also gives out rays of light in all direction. Stars, sun etc in universe are supposed to be spherical in shape.
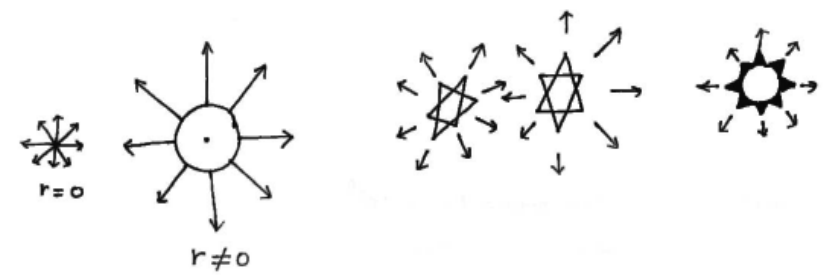

Fig 3: Point source, enlarged point source, star, sun etc. Let us consider a point source of light and the object be placed in the path of light which casts shadow on a screen. Since the source is point in form and size is smaller than object, only total shadow is casted on a screen. If the size of source equals to or greater than object, casts both total and partial shadows on a screen. That is why, to get only total shadow, source of light either in point form or in spherical form should be smaller than the size of opaque object.

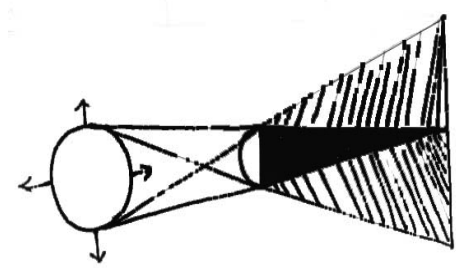

Fig 4: Formation of partial shadow due to greater size of source than object. 


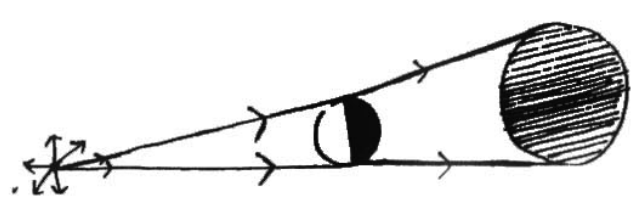

Fig 5: Formation of total shadow (darkest) by point source.

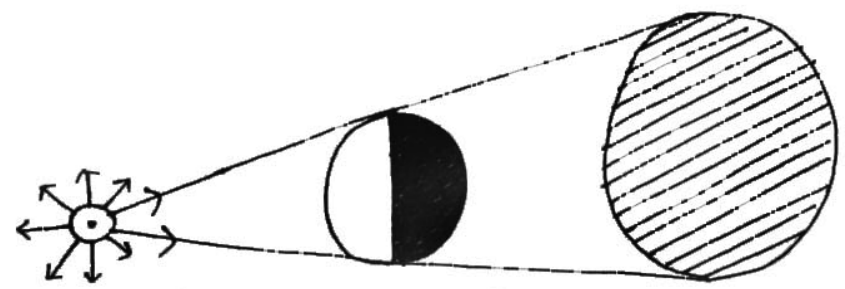

Fig 6: Formation of total shadow (sharp shadow) due to spherical source less than the size of object.

Let us consider the opaque sphere of radius $\mathrm{R}$. When light falls upon sphere, shadow is cast on screen. What we see is that: spherical object is of three dimensions. But cast two dimensional shadows. It means that frontal surface of sphere faced to source and unto which light falls is not responsible for casting shadow i.e. frontal curved surface having curved surface area $2 \pi R^{2}$ does not play any role to form 2D circular shadow on a screen but such $2 \mathrm{D}$ circular part or plane or surface, real or imaginary by geometry, exists in the object which plays role to produce shadow on a screen i.e. In the case of spherical object having radius $\mathrm{R}$, part or plane or $2 \mathrm{D}$ region that is responsible to caste exactly the corresponding shadow is cross sectional circle within sphere having area $\pi R^{2}$. Such an area of the object which plays role to cast corresponding shadow on a screen is termed as area of object. Denoted as ' $a$ '. e.g. when plane of square is taken, $a=1^{2}$. When plane of circle is taken, $a=\pi r^{2}$, when plane of triangle $t$ is taken, $\mathrm{a}=1 / 2 \mathrm{~b} \times \mathrm{h}$. When plane of irregular shaped object is taken, ' $a$ ' is calculated by graphical method. These planes object also caste the plane shadow with very similar shape to corresponding plane object.

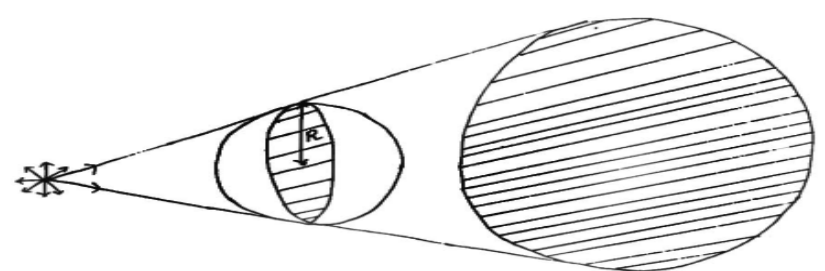

Fig 7: Sphere of radius R, showing the plane area within 3D sphere which casts shadow on a screen.

Curved surface has no role. Here, $a=\pi R^{2}$ but $a \neq 2 \pi R^{2}$, though $2 \pi R^{2}$ faces the source.

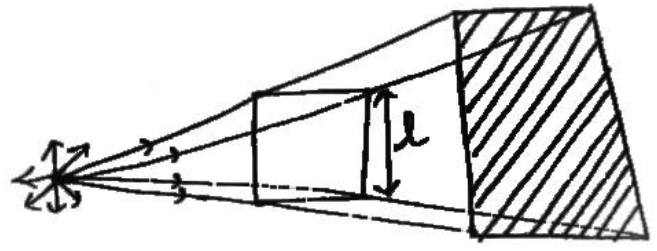

Fig 8: Square casting square shadow, $\mathrm{a}=1^{2}$

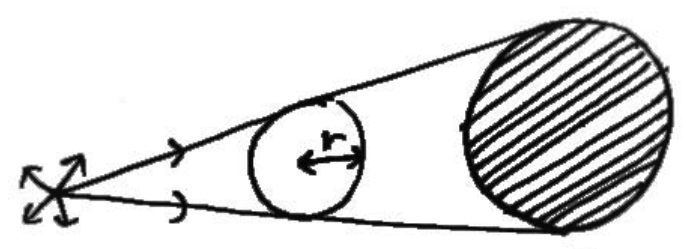

Fig 9: plane circle casting plane circular shadow $a=\pi r^{2}$.

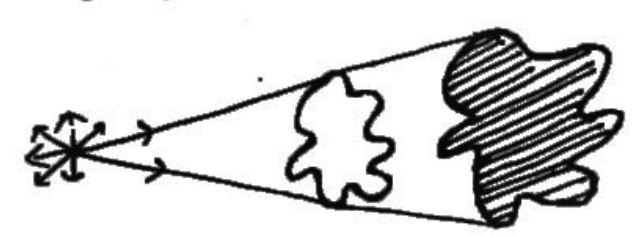

Fig10: irregular plane object. $\mathrm{a}=$ from graph.

As soon as light falls on an object, shadow appears on a plane surface of screen. So, naturally, there is need to place plane of screen parallel to the plane of object which is also known as area of object. So, plane of object i.e. area of object must be placed parallel to plane of screen

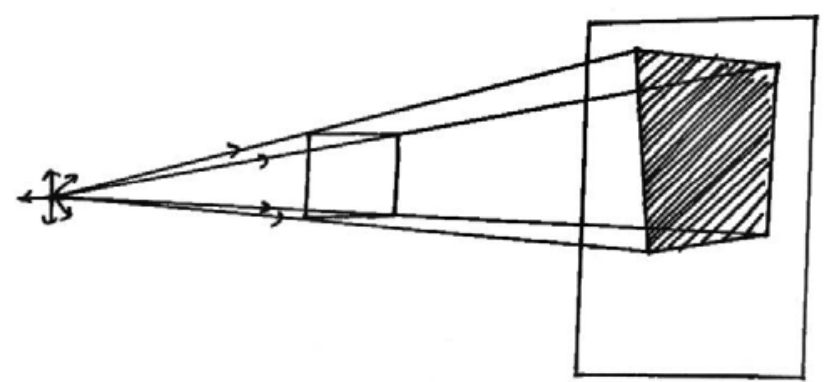

Fig 11: Arrangement of apparatus where screen is compulsory to be parallel with plane of object (area of object).

Let us discuss some technical terms.

Area of object - Discussed above. It is the responsible area of an object which form it's corresponding shadow on a screen. Denoted as ' $a$ '. For circle $a=\pi r^{2}$. Area of Shadow - Discussed above. It is the area of shadow which the given object caste on a screen Denoted as ' $A$ '. For circle, $A=\pi R^{2}$, where, $R$ is the radius of circular shadow of circular object.

Object distance - It is the normal distance between 
a point source or centre of spherical source and plane of object (area of object). Denoted as'd'.

Shadow distance - It is the normal distance between a point source or centre of spherical source and plane of screen (area of shadow). Denoted as ' $D$ '.

Object distance and shadow distance both are the portions of same normal ray through plane of object $\&$ screen.

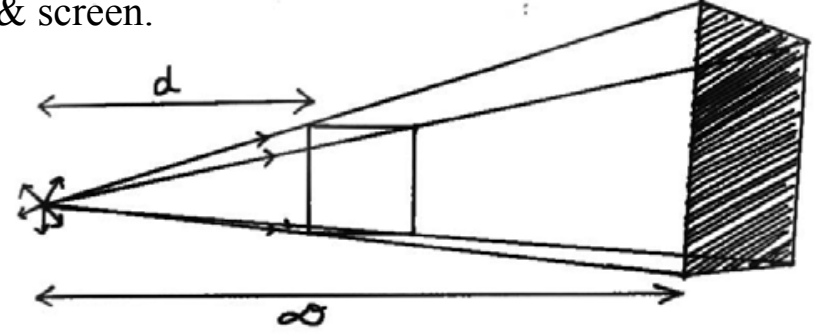

Fig 12: $\mathrm{d}$ is object distance as shown in fig. Similarly, D is shadow distance. Both distances are taken from point source or centre of spherical source, if spherical source is present.

\section{First law}

Shadow distance and object distance remaining constant. Area of shadow is directly proportional to the area of object. i.e. A $\alpha$ a

Where shadow distance and object distance remaining constant. Where, 'A' is area of shadow and ' $a$ ' is area of object.

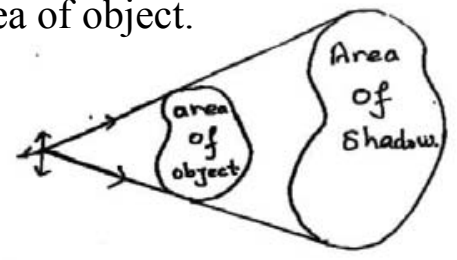

Keeping shadow distance and object distance constant, the object of area $a_{1}$ forms shadow of area $A_{1}$. Then it can be written as Ala1 $=$ Constant $=K$ Under the identical condition, the object of area $\mathrm{a}_{2}$ forms shadow of area $A_{2}$. Then it can be written as, $\mathrm{A} 2 \mathrm{a} 2=$ Constant $=\mathrm{K}$

So, under identical condition of shadow distance and object distance, we can write,

$$
\mathrm{A} 1 \mathrm{a} 1=\mathrm{A} 2 \mathrm{a} 2=\text { Anan }
$$

If circumference or perimeter is considered instead of area, this law hold true which can be written as, $\mathrm{C} 1 \mathrm{cl}=$ $\mathrm{C} 2 \mathrm{c} 2=\mathrm{Cncn}$ Where, $\mathrm{C}=$ circumferenceorperimeter of shadow. $\mathrm{c}=$ circumference or perimeter of object.

\section{Second law}

Area of object and object distance remaining constant, the area of shadow is directly proportional to the square of shadow distance. $A \alpha D^{2}$---- (2) where area of object and object distance are kept constant. ' $A$ ' is area of shadow and ' $D$ ' is shadow distance.

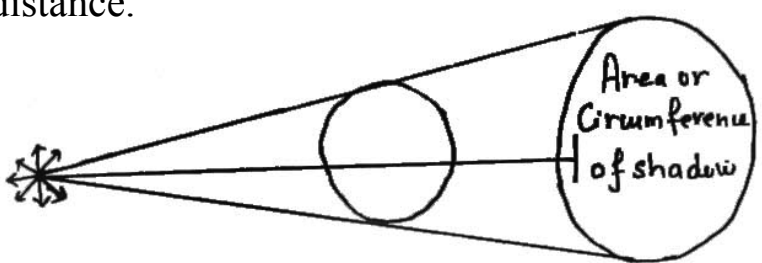

Shadow distance

Fig13: variables of $2^{\text {nd }}$ law

Keeping Area of object and object distance constant, shadow of area $A_{1}$ is formed at a shadow distance $D_{1}$. Then, A1D12 $=$ Constant $=\mathrm{K}$

Under the identical condition, shadow of area $\mathrm{A}_{2}$ is formed at a shadow distance $\mathrm{D}_{2}$. Then

$$
\mathrm{A} 2 \mathrm{D} 22=\text { Constant }=\mathrm{K}
$$

So, under identical condition of area of object and object distance, we can write,

$$
\mathrm{A} 1 \mathrm{D} 12=\mathrm{A} 2 \mathrm{D} 22=\mathrm{AnDn} 2 \text {. }
$$

If circumference or perimeter is considered instead of area, this law does not hold good. It becomes,
$\mathrm{C} 1 \mathrm{D} 1=$
$\mathrm{C} 2 \mathrm{D} 2=$
$\mathrm{CnDn}$

Where, $\mathrm{C}=$ Circumference or Perimeter of shadow.

$\mathrm{D}=$ Shadow distance

\section{Third law}

Shadow distance and area of object remaining constant. Area of shadow is inversely proportional to the square of object distance. i.e. A $\alpha 1 \mathrm{~d} 2$ (3) where shadow distance and area of object are kept constant. ' $\mathrm{A}$ ' is area of shadow and' $\mathrm{d}$ ' is object distance.

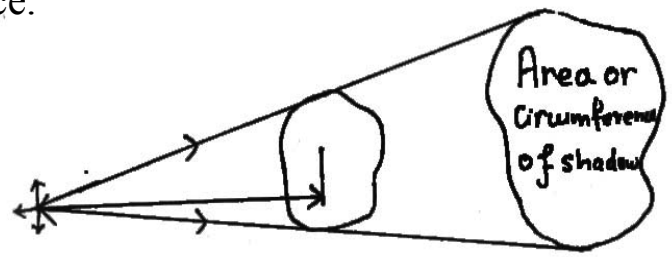

Object distance

Fig14: variables of $3^{\text {rd }}$ law

Keeping shadow distance and area of object constant, shadow of area $\mathrm{A}_{1}$ is formed at a object distance $\mathrm{d}_{1}$, then $\mathrm{A}_{1} \mathrm{~d}_{1}^{2}=$ Constant $=\mathrm{K}$

Under the identical condition, shadow of area $\mathrm{A}_{2}$ is formed at an object distance $\mathrm{d}_{2}$, then

$$
\mathrm{A}_{2} \mathrm{~d}_{2}{ }^{2}=\text { Constant }=\mathrm{K} \text {. }
$$

Under the identical condition of shadow distance 
and area of object, we can write,

$$
\mathrm{A}_{1} \mathrm{~d}_{1}{ }^{2}=\mathrm{A}_{2} \mathrm{~d}_{2}{ }^{2}=\mathrm{A}_{\mathrm{n}} \mathrm{d}_{\mathrm{n}}^{2}
$$

If circumference or perimeter is considered instead of area, above condition does not hold but becomes ... $\mathrm{C}_{1} \mathrm{~d}_{1=} \mathrm{C}_{2} \mathrm{~d}_{2=} \mathrm{C}_{\mathrm{n}} \mathrm{d}$ here, $\mathrm{C}=$ Circumference or Perimeter of shadow, $d=$ Object distance.

\section{Fourth law}

It is also known as combined law.

From first law, we have

A $\alpha$ a ---------- (1) Where shadow distance and object distance are constant.

From Second law, we have

$\mathrm{A} \alpha \mathrm{D}^{2}$ (2) Where area of object and object distance are constant.

From third law, we have
A $\alpha 1 / d^{2}$
(3) Where area of object and shadow distance are constant.

Combining eq ${ }^{\mathrm{n}}(1),(2),(3)$,

$\mathrm{A} \alpha \frac{a D^{2}}{d^{2}}$ i.e. none is Kept constant. All object and shadow area are variable.

Or, $\mathrm{Ad}^{2}=\mathrm{K} \cdot \mathrm{aD}^{2}$, Where $\mathrm{K}$ is constant. It is independent of all variables. For point source or spherical source, under described condition, value of constant $\mathrm{K}$ is always unity. i.e. $\mathrm{K}=1$.

$$
\text { Or, } \mathrm{Ad}^{2}=\mathrm{aD}^{2}
$$

this is the eq ${ }^{\mathrm{n}}$ which must be obeyed by nature in the process of forming shadow under developed condition. Formation of shadow of any shape can not violate $\mathrm{eq}^{\mathrm{n}}(4)$ where, $\mathrm{D}=$ shadow distance, $\mathrm{d}=$ object distance, $\mathrm{a}=$ area of object and $\mathrm{A}=$ area of shadow

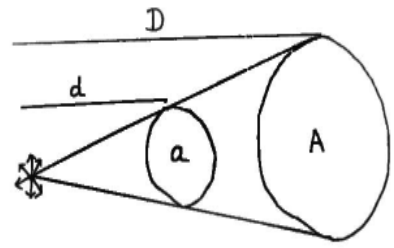

Fig15: formation of shadow goes through $\mathrm{aD}^{2}=\mathrm{Ad}^{2}$. I.e. always what happen is: area of object $\times$ square of shadow

distance $=$ area of shadow $\times$ square of object distance. If circumference or perimeter is considered, relation will be $\mathrm{Cd}=\mathrm{cD}$, where, ' $\mathrm{C}$ ' is Circumference or perimeter of shadow and ' $c$ ' is circumference or perimeter of object. So, when we want to know relation regarding area, rel $^{\mathrm{n}}$ is Area of shadow $\times$ square of object distance $=$ Area of object $\times$ square of shadow distance.

i.e. $\frac{d^{2}}{D^{2}}=$ unity

When we want to know relation regarding perimeter or circumference, relation is perimeter of shadow $\times$ object distance $=$ perimeter of object $\times$ shadow distance.

i.e. $\frac{d}{b}=$ unity

From (5) and (6), we can write,

$$
\frac{A d^{2}}{a D^{2}}=\frac{c D}{c D}
$$

Or, $\frac{A c}{a C}=\frac{d D^{2}}{d^{2} D} \quad$ or, $\frac{A c}{a C}=\frac{D}{d} \quad$ When both considered at a same time/condition.

i.e. Area of shadow $\times$ perimeter of object $=$ shadow distance $\times$ perimeter of shadow object distance.

\section{Verification:}

Point source is taken as the source of light. First of all, card board is taken and a very small hole is made in it. Electrical bulb is illuminated just behind the small pore of cardboard. This pore acts as a point source of light. Then circular disc of different sizes are taken as an object. Shadow distance and object distance are maintained constant. Then area of objects and corresponding area of shadows are also noticed.

Secondally, area of object and object distance are kept constant. The shadow distance is made different. The corresponding area of shadow of different shadow distances are noticed.

Thirdly, the shadow distance and the area of the object are kept constant. The object distance is made different. The corresponding area of shadows and object distances are noticed. The data obtained is as follows:- 


1st law:-
\begin{tabular}{|c|c|c|c|}
\hline $\mathrm{A}\left(\mathrm{cm}^{2}\right)$ & $\mathrm{a}\left(\mathrm{cm}^{2}\right)$ & $\mathrm{Aa}$ & Result \\
\hline 7.2 & 6 & 1.2 & \\
9.6 & 8 & 1.2 & \\
8.4 & 7 & 1.2 & $\mathrm{~A} \alpha \mathrm{a}$ \\
\hline
\end{tabular}

Hence, $1^{\text {st }}$ law is verified.

$2^{\text {nd }}$ law:-

\begin{tabular}{|c|c|c|c|c|}
\hline $\mathrm{A}\left(\mathrm{cm}^{2}\right)$ & $\mathrm{D}(\mathrm{cm})$ & $\mathrm{D}^{2}$ & $\mathrm{AD} 2$ & Result \\
\hline 36 & 3 & 9 & 4 & \\
16 & 2 & 4 & 4 & $\mathrm{~A} \alpha \mathrm{D}^{2}$ \\
100 & 5 & 25 & 4 & \\
\hline
\end{tabular}

$3^{\text {rd law:- }}$

Hence, $2^{\text {nd }}$ law is verified.

\begin{tabular}{|c|c|c|c|c|}
\hline$A\left(\mathrm{~cm}^{2}\right)$ & $d(\mathrm{~cm})$ & $\mathrm{d}^{2}$ & $A d^{2}$ & Result \\
\hline 22.95 & 8 & 64 & 1470 & \\
14.7 & 10 & 100 & 1470 & $A \alpha 1 \mathrm{~d} 2$ \\
30 & 7 & 49 & 1470 & \\
\hline
\end{tabular}

Hence, $3^{\text {rd }}$ law is verified.

\section{Results and Discussion:}

For the symmetrical point source:

1) The area of shadow varies in proportion to the area of object, keeping the shadow distance and object distance constant.

2) The area of shadow varies directly proportional to the square of shadow distance, keeping the area of object and object distance constant.

3) The area of shadow varies inversely proportional to the square of object distance, keeping shadow distance and area of object constant.

4) Collectively, the relationship among all can be expressed as a $\mathrm{D}^{2}=\mathrm{Ad}^{2}$.

Shadow is interesting part of nature carrying the mathematical virtue to guide it's self. From the study of 'Theory of shadow', we can describe the nature of shadow very easily. Sooner or later, many further research can be done over it.

\section{Conclusion}

The shadow is not simply a dark patch on a screen but the one which is governed by law of nature. The research in the field of shadow can lead to many discoveries.

\section{Reference:}

1) Science, grade 9, 2056. eclipse; Janak Shiksha Samagri Kendra, Kathmandu, Nepal.

2) S.K. Gupta and J.M.Pardhan 2006. A text book of physics; part- 1, Surya publications, Jawahar Nagar, Jalandhar India.

3) S.K. Gupta and J.M.Pardhan 2006. A text book of physics; part- II, Surya publications, Jawahar Nagar, Jalandhar India.

4) Kabi Raj Banatwa, Tika B. Katuwal, Ganapati N. Jha 2003. A text book of physics II, Ekta books Distributors Pvt.Ltd., Kathmandu, Nepal.

5) Earnest S.Greene, 1965. Principles of physics.

7) George O. Abell, $3^{\text {rd }}$ edition, 1964. Exploration of universe, pp154-158. 